\title{
La innovación evaluativa y el cambio de paradigma en la enseñanza contable
}

\section{Evaluative innovation and the paradigm shift in accounting education}

\author{
Merle Iglesias Mora* \\ merle@aeronationsa.com \\ Pedro Iglesias Mora** \\ piglesias@uteg.edu.ec
}

\begin{abstract}
Resumen
El objetivo de la investigación consistió en analizar el sistema de evaluación actual de la asignatura de Contabilidad en el área empresarial, para determinar su incidencia en el rendimiento académico de los estudiantes. El nivel de la investigación fue exploratorio y descriptivo, ya que pretendió precisar la situación investigada sin contar con antecedentes informativos previos al estudio; por su diseño se consideró de campo, basada en la recopilación de datos cuantitativos. Los resultados obtenidos permitieron proponer el diseño de nuevas estrategias de evaluación de los saberes, considerando los aspectos elementales de la evaluación educativa determinados por la Autoridad Nacional y las experiencias logradas por los docentes de las instituciones educativas.
\end{abstract}

\section{Palabras clave}

Estrategias, rendimiento académico, resultados de aprendizaje, sistema de evaluación.

* Ecuatoriana. Máster en Administración de Empresas. Máster en Diseño y Evaluación de Modelos Educativos. Universidad Tecnológica Empresarial de Guayaquil- UTEG. Docente de la cátedra Contabilidad y Tributación.

* Ecuatoriano. Candidato a Doctor en Gestión Económica Global. Magister en Administración de Empresas. Universidad Tecnológica Empresarial de Guayaquil- UTEG. Director Administrativo, docente de la Modalidad Semipresencial de Posgrado. 


\section{Abstract}

The objective of the investigation was to analyze the current evaluation system of the subject of Accounting in the business area, to determine its incidence in the academic performance of the students. The level of the investigation was exploratory and descriptive, since it tried to specify the situation investigated without having previous informative antecedents to the study; for its design was considered of field, based on the collection of quantitative data. The results obtained allowed to propose the design of new strategies for the evaluation of knowledge, considering the elementary aspects of the educational evaluation determined by the National Authority and the experiences achieved by the teachers of educational institutions.

\section{Keywords}

Academic performance, evaluation system, learning results, strategies.

Forma sugerida de citar: Iglesias Mora, Merle \& Iglesias Mora, Pedro (2016). La innovación evaluativa y el cambio de paradigma en la enseñanza contable. Retos, 12(2), pp. 165-178

\section{Introducción}

En el contexto de nuestra educación, y en concordancia con la innovación curricular vigente, estas estrategias deben diversificarse y cumplir con lo descrito por la Autoridad Nacional para la evaluación educativa, quien la define en el artículo 184 del Reglamento General a la LOEI ${ }^{1}$ como "... un proceso continuo de observación, valoración y registro de información que evidencia el logro de objetivos de aprendizaje de los estudiantes y que incluye sistemas de retroalimentación, dirigidos a mejorar la metodología de enseñanza y los resultados de aprendizaje" (Ministerio de Educación del Ecuador, 2016).

1 Ley Orgánica de Educación Intercultural del Ecuador.
Entre las características principales que debe tener la evaluación educativa en general, se considera también como algo primordial el que ésta sea científica, continua y "sistemática integrada dentro del proceso educativo" (Rosales, 2014, p. 2). Justamente el hecho de ser científica, obliga a que las nuevas formas de evaluación, estén basadas en técnicas e instrumentos adecuados a los distintos grupos, momentos y naturaleza de las destrezas evaluadas, ya que "la evaluación no debe verse como una acción unilateral y terminal por parte del docente, sino como una práctica que va a guiar su enseñanza..." (Torres, 2012, p. 4).

A pesar del fácil acceso a la información que existe en la actua- 
lidad los recursos disponibles son ignorados por muchos de los docentes. Pero ¿por qué tratar sobre la metodología, si lo que nos compete es la evaluación?, como sabemos, en el campo de la pedagogía es imposible hablar de coherencia curricular, si no se establece una relación adecuada entre los métodos de enseñanza, las técnicas y los instrumentos de valoración de los saberes.

\section{Figura 1. Proceso de construcción de resultados de aprendizaje} para una asignatura
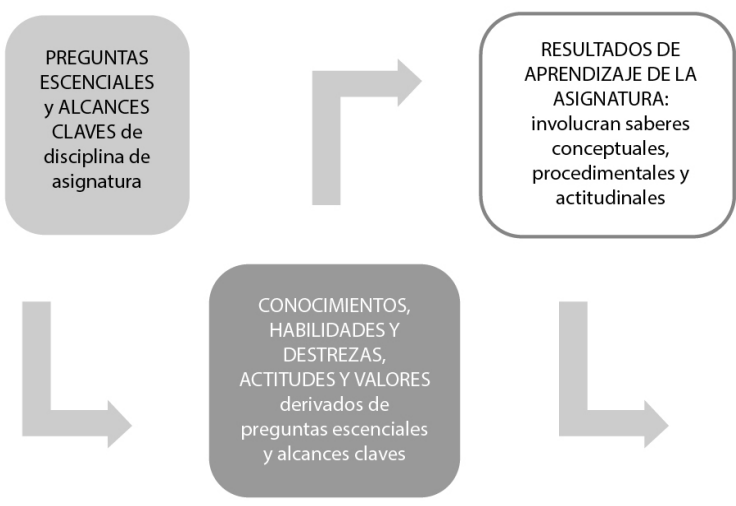

Fuente: Unidad de Gestión Curricular (UGC), UDLA.

En lo referente a la evaluación de asignaturas del área empresarial, surge la problemática de que aunque algunos docentes en ciertas instituciones han utilizado en la práctica evaluaciones de tipo innovador, la gran mayoría sigue utilizando la evaluación escrita como única técnica, perdiendo de esta manera la concordancia lógica que debe existir en el desarrollo de conocimientos contables y financieros útiles para la resolución de problemas vinculados con la economía actual, buscando desarrollar en los estudiantes destrezas como anali- zar, descubrir, elaborar hipótesis, confrontar, reflexionar, argumentar y comunicar ideas (Del Valle \& Curotto, 2008) y una "apropiación creativa de los conocimientos por parte de los estudiantes" (Ortiz, 2012, p. 36) que es hacia donde deben ser enfocados los aprendizajes y por ende la evaluación, debido a la naturaleza práctica de la asignatura y su orientación hacia el desenvolvimiento en el mundo comercial.

Aún existe el terrible error de algunos maestros al confundir evaluación con calificación, siendo estos términos muy relacionados 
entre sí pero no iguales, como indican pertinentemente Castillo \& Cabrerizo (2010): ..."el proceso evaluador es mucho más amplio y mucho más complejo que efectuar una simple medición, por lo que los datos aportados por la medición han de ser interpretados a la hora de evaluar" (p. 24). Esto recalca el hecho de que en los tiempos actuales la evaluación debe ser integral y basada en objetivos propuestos e indicadores de evaluación acordes a los procesos de enseñanza.

Es en virtud de todo lo descrito que se formula como interrogante el problema de investigación: ¿Es adecuado el sistema de evaluación que aplican a los estudiantes en la asignatura de Contabilidad del área empresarial algunas instituciones educativas de la ciudad de Guayaquil?

Mediante este trabajo se plantea implementar un nuevo sistema de evaluación tendiente a mejorar el perfil de salida de los estudiantes, diseñando a la vez un modelo que contemple "una variedad de modalidades de evaluación, lo que además tiene una connotación importante para la validez y confiabilidad del proceso evaluativo" (MINEDUC Chile, 2013, p. 10) y que a la vez por su generalidad pueda ser aplicado en otras instituciones que oferten en su malla curricular asignaturas similares.
El estudio permite a través del muestreo conocer las apreciaciones de los estudiantes pertenecientes a tres instituciones educativas de la ciudad de Guayaquil, que ofertan las especialidades del área empresarial, quienes consideraron que no son evaluados cualitativa y cuantitativamente en todos los saberes y además que los tiempos son muy prolongados en la realización de las evaluaciones. Es por esto que se ha desarrollado un plan que permita aprovechar cada instancia de evaluación estableciendo diversas estrategias, que satisfagan las necesidades de los estudiantes y a la vez los orienten "a pensar en la manera en que aprenden desarrollando en ellos una autosupervisión y una autoconciencia reflexivas" (Woolfolk, 2010, p. 123).

A través de la percepción docente de los investigadores y otros colaboradores consultados, se ha descubierto como problemática en la asignatura, que aunque los métodos empleados para los procesos de enseñanza, son acordes a las características técnicas y prácticas de la materia, los procesos evaluativos dejan mucho que desear en lo referente a la diversificación de técnicas e instrumentos apropiados ya que no "considera diversos factores como las diferencias individuales, los intereses y necesidades educativas especiales de los estudiantes..." (Ministerio de Educación del Ecuador, 2016). 
Surge también a través del estudio la imperiosa necesidad de que los estudiantes sean atendidos de manera personalizada; es por esto que como estrategia principal se establece la atención inmediata y la recuperación pedagógica continua, por medio de acciones como: preparar actividades sobre un mismo contenido y/o destrezas de repaso, de refuerzo y de profundización variando el grado de dificultad y el tipo de procesos que se ponen en juego (Subsecretaría de Fundamentos Educativos, MINEDUC, 2016), de manera que no se espera a que el estudiante baje su rendimiento e incluso fracase en su vida estudiantil para tomar acciones tendientes al afianzamiento del aprendizaje "buscando la adaptación a las necesidades individuales, y en la creación de vínculo entre educadores y alumnos para favorecer un acompañamiento personal eficaz" (Longás, Civís, \& Riera, 2013, p. 110) y por ende el mejoramiento de los resultados académicos.

¿Cómo se va a lograr esto? La evaluación estará diseñada para evaluar los cuatro pilares de la educación enunciados en el informe a la UNESCO, aprender a: conocer, hacer, vivir juntos y ser (Delors, 2012); en todas las facetas de la enseñanza de la Contabilidad a los estudiantes de bachillerato en ciencias con mención en Gestión Em- presarial y esta se va a concentrar en tres ejes: uno es la evaluación en clases incluyendo técnicas innovadoras de trabajo colaborativo como talleres prácticos de resolución de problemas y análisis de casos, sistematizadas en forma conjunta con las tradicionales formas individuales de evaluación como lecciones escritas, actuaciones en clase y desarrollo de guías de investigación.

El uso coordinado del aprendizaje autónomo y el aprendizaje colaborativo permitirá desarrollar competencias y cualidades importantes para la formación integral de los estudiantes. "En el aprendizaje colaborativo el estudiante es responsable de su proceso con el respaldo de sus compañeros, permite abrir perspectivas pues todas las posturas son tomadas en cuenta, por lo tanto promueve el respeto" (Crispín, 2011, p. 62).

Otro eje es la evaluación de las actividades desarrolladas en casa como tareas de resolución de ejercicios, trabajos de investigación y por supuesto los exámenes bimestrales; procesos que serán guiados transversalmente por el desarrollo de valores como la colaboración y el respeto en el trabajo de equipo, generando cambios de actitud, además de la posibilidad de asumir responsabilidades y compromisos con una tarea (Beltrán, Pereira, \& Sáez, 2011). 
Figura 2. Modelo de estrategias evaluativas

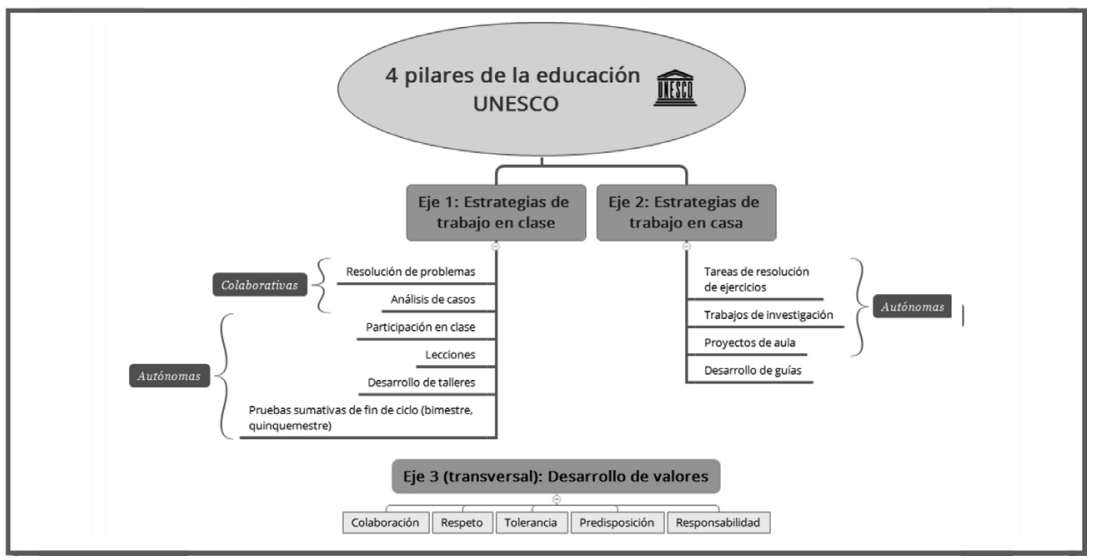

Fuente: Elaboración propia.

La naturaleza del presente estudio, acarrea beneficios para toda la comunidad educativa, es decir, tanto para directivos, como para docentes, padres de familia, sociedad en general y por supuesto para los estudiantes, que son la razón de ser de toda institución educativa. Existe la disposición para aceptar el reto de cubrir esa necesidad, así se obtendrá un beneficio en conjunto desarrollando jóvenes que apliquen las habilidades del pensamiento como la observación, establecen características, relacionan, buscan semejanzas y diferencias (OEI- MINEDUC Perú, 2014), comparan y por supuesto analizan para que de manera argumentada, puedan dar su apreciación e incluso puedan llegar a proponer mejoras tanto a nivel de los sistemas tecnológicos contables como en la manera de realizar los procedimientos y, con una formación en valores.

Los docentes estarán en capacidad de formular una diversidad de criterios y aplicar técnicas e instrumentos de evaluación acordes a cada desempeño auténtico diseñado en clase, los cuales guiarán el desarrollo de los procesos, recordando el hecho de que un cambio en la evaluación, exige a la vez el desarrollo de mejores propuestas en lo referente a las estrategias metodológicas de aula postuladas en las planificaciones meso y microcurriculares (Tobón, 2013).

El beneficio para la institución es obvio, ya que al mejorar la calidad del desempeño docente, se potencian los aprendizajes de los estudiantes, creando una estabilidad emocional, autoestima y mejores rendimientos que permiten crecer 
a la institución en sus componentes académico, humano y económico.

\section{Metodología}

El nivel de la investigación es exploratorio y descriptivo, ya que en lo referente a la evaluación de procesos en asignaturas del área contable a nivel medio, no existen registros en Ecuador de estudios precedentes, a pesar de ello cabe recalcar que existen evidencias de proyectos de tesis, que proponen estrategias evaluativas para la asignatura en algunas instituciones de nivel superior. La investigación "busca especificar las propiedades y pretende describir el fenómeno a través de las características observadas" (Hernández, Fernández, \& Baptista, 2010, p. 80), el comportamiento de los datos y la relación entre las variables para reconocer cuál es la incidencia del sistema de evaluación en el rendimiento académico y la promoción de los estudiantes en la asignatura de Contabilidad.

Por su diseño el estudio se considera de campo, ya que manipula una o más variables (Hernández, Fernández, \& Baptista, 2010) basado en la recolección de datos cuantitativos y cualitativos, tanto ex post facto como correlacionales, buscando el análisis a través de métodos generales como el InductivoDeductivo, el Analítico-Sintético y el método Estadístico para la tabu- lación e interpretación de los datos que conforman la muestra.

En la primera etapa de la investigación se elaboró un marco teórico por medio de fuentes secundarias como libros, revistas especializadas, videos, consultas en bases de datos de Internet y el apoyo de documentos sobre estudios cualitativos y cuantitativos relacionados con el tema. Esto permitió la operacionalización de las variables, y el desprendimiento de las dimensiones e indicadores que establecen la línea pertinente para la investigación.

La segunda etapa de la investigación consistió en la recolección de información de fuentes primarias como la secretaría de las instituciones educativas. Se seleccionó información referente a las calificaciones obtenidas en el último año, las cuales se organizaron por actividad, número de estudiantes y años de estudio, para así determinar los tres casos prácticos más exitosos y de esta manera poder implementar el proyecto.

El levantamiento de información se realizó a través de encuestas y entrevistas en profundidad aplicadas a los directivos, docentes de la asignatura y otros miembros de la comunidad educativa; además, se estudió en la medida de lo posible, los registros académicos de los tres procesos anuales anteriores, en busca de documentos de tipo académico como planes operativos, 
planificaciones microcurriculares y formatos de evaluaciones, las cuales nos brindan información de primer orden sobre el manejo que se le dio a las evaluaciones y su incidencia en las calificaciones registradas.

La población objeto de estudio estuvo conformada por 273 estudiantes de segundo de bachillerato del área Empresarial o Comercio de tres instituciones educativas de la ciudad de Guayaquil durante el año lectivo 2014-2015.

La muestra determinada fue de 160 estudiantes seleccionados por muestreo aleatorio simple. Una vez determinada la muestra se recolectó información por medio de una encuesta de base estructurada, procediéndose luego al uso de los métodos descriptivos para el análisis de la información, los cuales permitieron contrastar los datos y establecer porcentajes de aceptación o rechazo respecto a los procesos evaluativos que se utilizan en las diversas instituciones educativas.

\section{Resultados}

Los resultados que se muestran a continuación fueron obtenidos de la implementación de encuestas a los estudiantes de segundo de $\mathrm{Ba}$ chillerato de distintas Instituciones educativas que ofertan la opción empresarial, conformando el número de la muestra a través de la selección equitativa pero aleatoria de los participantes por paralelos.

La selección de las preguntas se realizó con base en el estudio de la información obtenida de fuentes escritas que precedió a la toma de datos; la estructura del cuestionario, por tanto no fue elaborada con base en la escala de Likert como se acostumbra en estos casos, sino estableciendo parámetros delimitados que permitieronn a los estudiantes seleccionar más puntualmente sus apreciaciones sobre las técnicas e instrumentos de evaluación y las características que estas deberían tener.

Como los estudiantes tenían la posibilidad de marcar más de una opción, las frecuencias anotadas no necesariamente suman el total de la muestra, sino más bien nos presentan una visión general de las tendencias marcadas entre los alumnos acerca de las formas de evaluar, obteniéndose los siguientes resultados:

Tabla 1. Evaluación de las pruebas de Contabilidad

Pregunta $\mathrm{N}^{\circ} 1$ ¿ ¿Cómo quisieras que te evalúen en las pruebas de Contabilidad?

\begin{tabular}{|l|l|l|}
\hline 1. & Que se valore solo el resultado & 46 \\
\hline 2. & Que se valore solo procedimiento & 112 \\
\hline 3. & Que se valoren ambos por igual & 125 \\
\hline
\end{tabular}

Fuente: Elaboración de los autores 
Gráfico 1. Evaluación de las pruebas de Contabilidad

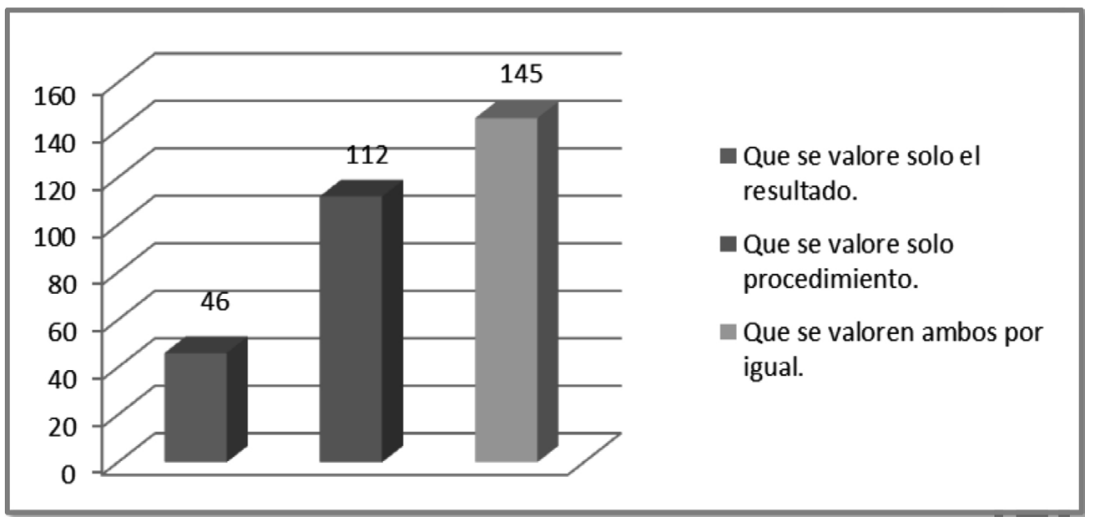

Fuente: Elaboración propia

Tabla 2. Otras formas de Evaluación

Pregunta $N^{\circ} 2$ ¿ ¿Además del examen que te gustaría que te evalúen?

\begin{tabular}{|l|l|l|}
\hline 1. & Deberes & 79 \\
\hline 2. & Talleres en clase & 103 \\
\hline 3. & Proyectos & 85 \\
\hline
\end{tabular}

Fuente: Elaboración de los autores.

Gráfico 2. Otras formas de Evaluación

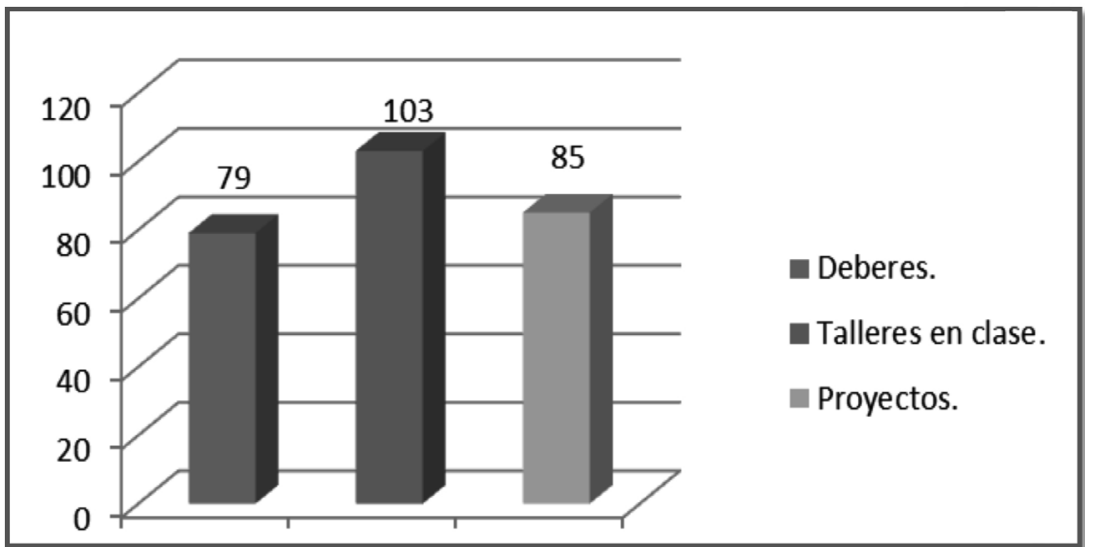

Fuente: Elaboración propia 
Tabla 3. Aplicación de evaluación periódica

\begin{tabular}{|l|l|l|}
\hline \multicolumn{3}{|c|}{ Pregunta No 3. Las evaluaciones deberian ser: } \\
\hline 1. & Semanales & 109 \\
\hline 2. & Al final de cada unidad & 77 \\
\hline 3. & Solo al final del proceso & 55 \\
\hline
\end{tabular}

\section{Gráfico 3. Aplicación de evaluación periódica}

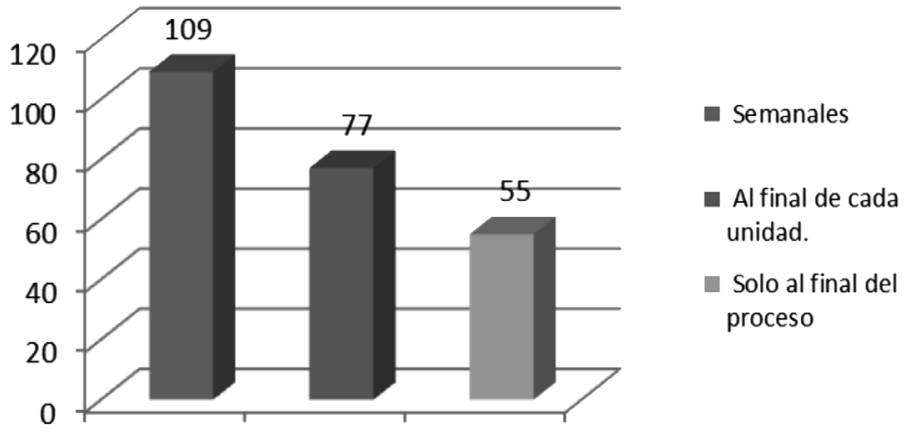

Fuente: Elaboración propia

Tabla 4. Aplicación de estrategias innovadoras

Pregunta $N^{\circ} 4$. ¿Cuáles de estas estrategias de aprendizaje considera que son innovadoras y deberían aplicarse en la clase?

\begin{tabular}{|l|l|l|}
\hline 1. & Aprendizaje basado en problemas & 88 \\
\hline 2. & Análisis de casos & 93 \\
\hline 3. & Ferias empresariales & 105 \\
\hline
\end{tabular}

Fuente: Elaboración de los autores con información del levantamiento 
Gráfico 4. Aplicación de estrategias innovadoras

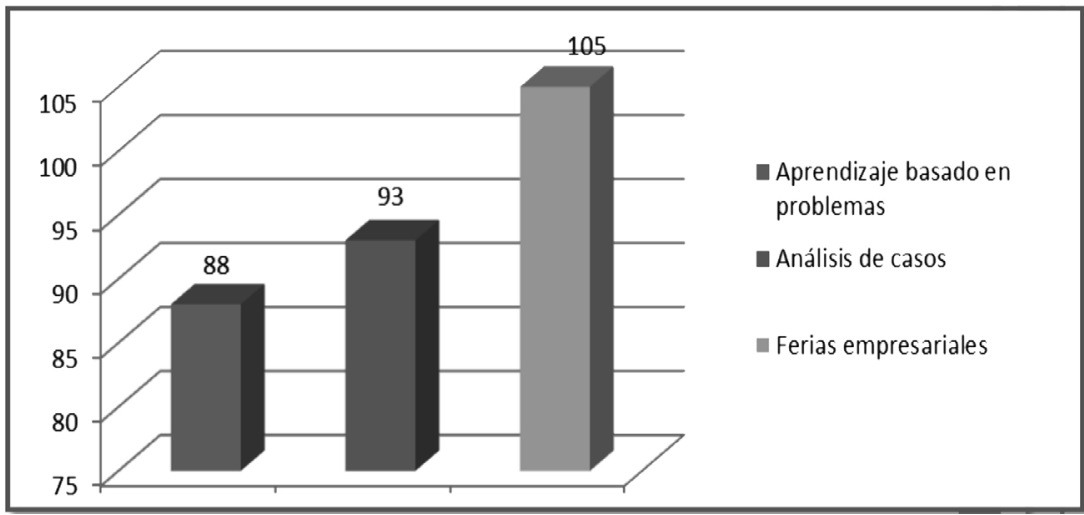

Fuente: Elaboración propia

Como parte de la investigación, se constataron también algunos documentos correspondientes a los PCI de las instituciones que nos brindaron su colaboración, como los planes anuales y de clase, en los que se puede constatar el uso de técnicas poco variadas, así como el bajo nivel de logro adquirido en las pruebas de tipo sumativo, a pesar de que en los parámetros de la evaluación formativa, los niveles no se encuentran tan bajos.
Como parte del acuerdo de confidencialidad establecido con las Instituciones colaboradoras, no es posible presentar los cuadros de rendimiento de los estudiantes en los dos períodos anteriores, pero se trabajó una estadística acerca del uso de técnicas e instrumentos de evaluación con base en la revisión de los planes académicos de los maestros pertenecientes al área de comercio o empresariales, a la que pertenece la asignatura de Contabilidad. Los resultados fueron los siguientes:

Tabla 5. Uso de técnicas e instrumentos de evaluación

\begin{tabular}{|l|l|l|}
\hline \multicolumn{1}{|c|}{ Técnica } & \multicolumn{1}{|c|}{ Instrumento } & \multicolumn{1}{c|}{$\%$ de uso } \\
\hline \multirow{2}{*}{ Prueba escrita } & Cuestionario semiestructurado & 100,00 \\
\cline { 2 - 3 } & Cuestionario de base estructurada & 71,43 \\
\hline Pruebas orales y de actuación & Lista de cotejo & 0,00 \\
\hline Talleres grupales & Escala valorativa & 0,00 \\
\hline Exposiciones y Proyectos & Rúbrica & 42,86 \\
\hline
\end{tabular}


Gráfico 5. Uso de técnicas e instrumentos de evaluación

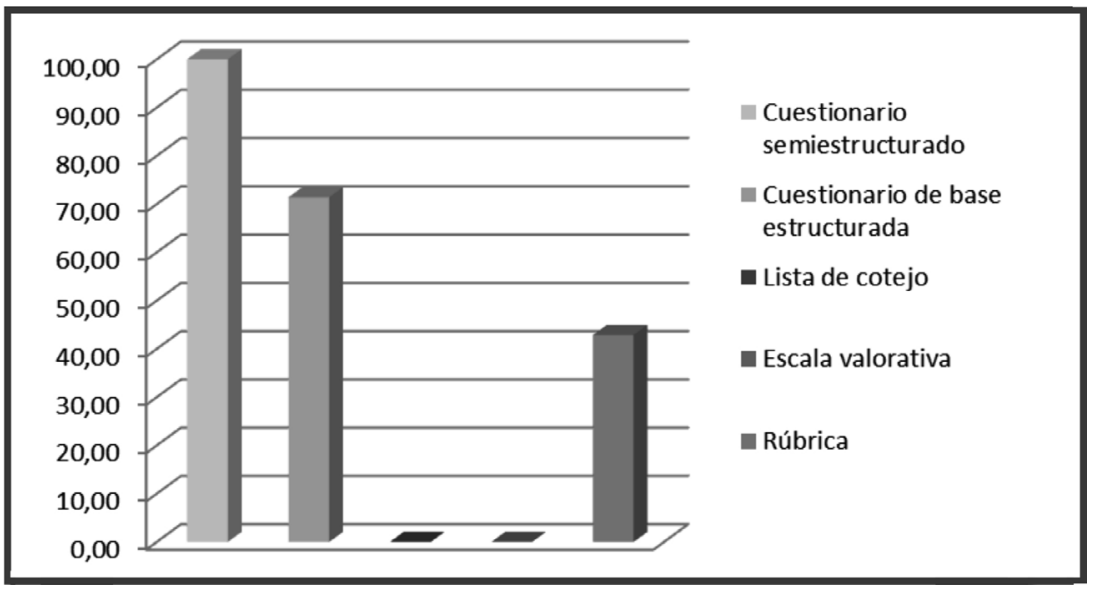

Fuente: Elaboración propia

\section{Discusión}

Los resultados evidencian un alto interés por parte de los estudiantes, en los procesos de cambio e innovación evaluativa, lo cual exige una mejor preparación y planificación por parte de los docentes.

Los altos porcentajes obtenidos en las dos primeras preguntas permiten determinar la necesidad de realizar evaluaciones enfocadas no solamente en la calificación $(62,5 \%)$, sino que permitan una valoración cuanti-cualitativa de los aprendizajes $(90,63 \%)$ y a la vez el establecimiento de planes de recuperación pedagógica. La falta de variabilidad en los instrumentos utilizados se puede corroborar en la tercera pregunta, mientras que la cuarta nos indica el interés de los estudiantes por una propuesta evaluativa más continua $(68,13 \%)$ y que no solamente se considere evaluar al final de los procesos.

Otros estudios similares (Mateos-Ronco, Lajara-Camilleri, \& Marín Sánchez, 2011) consideran con base en sus resultados a la evaluación continua y la realimentación como procesos efectivos.

Los métodos de enseñanza basados en la construcción del conocimiento práctico, como son el Aprendizaje basado en problemas, el Análisis de casos y los proyectos de Ferias empresariales, son también acogidos con gran entusiasmo, ya que en el campo educativo, los procesos de aprendizaje, deben ser concordantes con las técnicas e instrumentos utilizados para la evaluación. 


\section{Conclusiones}

En la investigación de campo realizada, se pudo constatar que el sistema de evaluación que se viene aplicando en el área empresarial de algunas instituciones educativas de Guayaquil, está incidiendo en el rendimiento de los estudiantes, esto se muestra claramente por medio de las apreciaciones de los estudiantes así como también en los registros de rendimiento que reposan en la secretaría de los planteles.

En atención a las encuestas aplicadas se pudo constatar que los métodos de evaluación no son holísticos e integradores en la elaboración de exámenes de grado.

La falta de estrategias pedagógicas innovadoras y la repetitividad de los sistemas tradicionales de evaluación se denotan tanto en los registros de planificación como en las estadísticas obtenidas, causando desmotivación en los estudiantes y por ende incidiendo negativamente en el rendimiento académico de los mismos.

En atención a la investigación realizada se concluye la necesidad de que se realicen cambios en el sistema de evaluación y en tal sentido se plantea el diseño y aplicación de un manual de procesos para la evaluación.

\section{Bibliografía}

Beltrán, J., Pereira, J., \& Sáez, J. (2011). Aplicación práctica de técnicas docentes para Contabilidad Financiera. Educade, 3-34.
Castillo, S., \& Cabrerizo, J. (2010). Evaluación educativa de aprendizajes y competencias. Madrid: Pearson.

Crispín, M. L. (2011). Aprendizaje autónomo. Orientaciones para la docencia. México DF: Universidad Iberoamericana.

Del Valle, M., \& Curotto, M. (2008). La resolución de problemas como estrategia de enseñanza y aprendizaje. Revista Electrónica de Enseñanza de las Ciencias, 463-479.

Delors, J. (2012). La educación encierra un tesoro. Informe a la UNESCO. Paris: UNESCO.

Hernández, R., Fernández, C., \& Baptista, M. (2010). Metodología de la Investigación. México: Mc Graw Hill.

Longás, J., Civís, M., \& Riera, J. (2013). Refuerzo escolar e inclusión educativa: propuesta teórico-práctica a partir de la experiencia de apoyo al éxito escolar del programa CaixaProinfancia. Revista de Educación Inclusiva, 106-124.

Mateos-Ronco, A., Lajara-Camilleri, N., \& Marín Sánchez, M. (2011). Sistema de evaluación en contabilidad y resultados. Obtenido de http://web.ua.es/es/ice/jornadasredes-2011/documentos/posters/185422.pdf

MINEDUC Chile (2013). Evaluación para el Aprendizaje en CCNN. Santiago.

Ministerio de Educación del Ecuador (2016). Reglamento a la LOEI. Quito: Dirección Nacional de Normativa Jurídico Educativa.

OEI - MINEDUC Perú (2014). 15 Buenas prácticas docentes. Lima: Prysma S.A.C.

Ortiz, A. (2012). Metodología de la ense- 
ñanza problémica en el aula de clases (Segunda ed.). Barranquilla: ASIESCA.

Rosales, M. (2014). Proceso evaluativo: evaluación sumativa, evaluación formativa y Assesment su impacto en la educación actual. En: Congreso Iberoamericano de Ciencia, Tecnología, Innovación y Educación. Buenos Aires.

Subsecretaría de Fundamentos Educativos, MINEDUC (2016). Instructivo para la aplicación de la evalua- ción estudiantil. Quito.

Tobón, S. (2013). Formación integral y competencias: Pensamiento complejo, diseño curricular, didáctica y evaluación. Bogotá: Ecoe Ediciones.

Torres, R. (2012). La evaluación de los aprendizajes en el contexto de la atención a las necesidades educativas de los estudiantes. San José: Equipo Editorial CENAREC.

Woolfolk, A. (2010). Psicología Educativa. México: Pearson Educación.

Recepción: 09- 08- 2016 - Aceptación: 11-26-2016. 